\title{
Prediction of SiO2 Nano CoAting Properties Using FuzzY LogiC
}

\author{
Antons Stekleins, Eriks Gerins \& Artis Kromanis
}
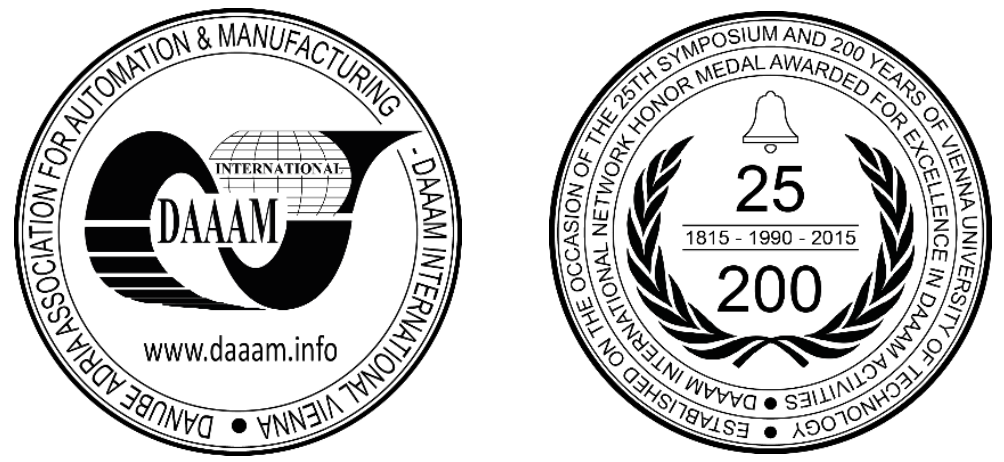

This Publication has to be referred as: Stekleins, A[ntons]; Gerins, E[riks] \& Kromanis, A[rtis] (2017). Prediction of SiO2 Nano Coating Properties Using Fuzzy Logic, Proceedings of the 28th DAAAM International Symposium, pp.06540660, B. Katalinic (Ed.), Published by DAAAM International, ISBN 978-3-902734-11-2, ISSN 1726-9679, Vienna, Austria

DOI: $10.2507 / 28$ th.daaam.proceedings.092

\begin{abstract}
The paper analyses $\mathrm{SiO} 2$ vacuum nano coating technological process and its influence on coating quality and properties. Use of fuzzy logic is proposed to develop a process prediction models for vacuum coating. Several experiments were designed and conducted using UV80 laboratory vacuum system, wherein SiO2 coating light transmission coefficient and thickness were measured. $\mathrm{SiO} 2$ nano coating light transmission and thickness predictions models were designed using fuzzy logic techniques. After preliminary analysis it was concluded that models developed by fuzzy logic have high accuracy and can be used to predict $\mathrm{SiO} 2$ vacuum nano coating properties if a leak in vacuum system appears during technological process, thus enabling continuation of the technological process as long as coating properties will match selected criteria.
\end{abstract}

Keywords: SiO2; vacuum coating; fuzzy logic; prediction model; coating deposition

\section{Introduction}

Nowadays nano coatings are one of the most commonly used and desired product in vacuum field, which can be applied onto webs, films and foils. For example, in $\mathrm{SiO} 2$ vacuum nano coating technology a magnetron sputtering is used and $\mathrm{SiO} 2$ coating itself is unique and demanded coating due to its properties. Thin layers having high insulation resistance are required for many applications in electronics and in sensor and photovoltaic technology [2]. $\mathrm{SiO} 2$ is one of these materials. These applications include gate oxide layers in microelectronics (which are only a few nanometers thick), insulating layers in sensor applications (for which higher dielectric strengths can be required) and insulating layers in photovoltaic technology (for which the requirements on dielectric strength are lower) [2]. Therefore, it is essential to maintain required coating technological process parameters to provide good product quality. System leakage is one of the main problems we encountered with during $\mathrm{SiO} 2$ coating process. Pressure deviations, process parameter changes and leakage can damage the coating, its quality and properties. Therefore, it is necessary to establish models, which can be used to predict vacuum nano coating properties when there is a leakage in vacuum system. Such models would help to evaluate $\mathrm{SiO} 2$ coating sputtering process condition and predict its output parameters (light transmission coefficient and 
thickness). In this study models were developed using fuzzy logic method. Fuzzy set theory which formed the basis of fuzzy logic, as an alternative to classical set theory, was presented by L.A. Zadeh [1]. In the fuzzy set theory, the transition between membership to non-membership is done progressively [1].

Unlike Boolean Logic fuzzy logic was designed to reproduce human thinking technique using mathematical approach $[1,5]$. This particular approach helps to visualize and predict possible output data variables depending on the input data changes $[3,7,6]$. Several $\mathrm{SiO} 2$ experiments were designed and conducted, a light transmission coefficient and thickness were measured for each coating sample. Using fuzzy logic method thickness and light transmission coefficient models were developed and compared with measured values.

\section{Design of SiO2 coating experiment}

Experiments to develop prediction models were carried out at a laboratory vacuum machine UV80 for coating webs and films [8]. Silicon reactive sputtering was selected for the experiments, where $\mathrm{SiO} 2$ coating was applied onto polyethylene terephthalate (PET) film. For the process power supply $(8 \mathrm{~kW})$, argon flow $75 \mathrm{sccm}$, variable oxygen and airflows were used. Main idea of the experiment was to change airflow quantity thus simulating leak condition, to verify coating quality, i.e. measure its thickness and a light transmission coefficient. After conducting SiO2 vacuum coating experiments samples were verified and measured. Measured results are given in table 1.

\begin{tabular}{|c|c|c|c|c|}
\hline No. & O2, sccm & Air, sccm & $\begin{array}{c}\text { d } \\
\text { (thickness, } \mathbf{~ n m})\end{array}$ & $\begin{array}{c}\text { T } \\
\text { (transmission coefficient, \%) }\end{array}$ \\
\hline 1 & 0 & 70 & 142.1 & 86.64 \\
\hline 2 & 0 & 65 & 133 & 86.49 \\
\hline 3 & 0 & 55 & 128.5 & 87.54 \\
\hline 4 & 0 & 50 & 121.3 & 88.36 \\
\hline 5 & 7 & 45 & 115.2 & 89.35 \\
\hline 6 & 17 & 40 & 110.7 & 89.45 \\
\hline 7 & 20 & 35 & 109.8 & 89.19 \\
\hline 8 & 25 & 30 & 112.8 & 87.66 \\
\hline 9 & 30 & 25 & 11.6 & 87.55 \\
\hline 10 & 41 & 15 & 119.9 & 86.81 \\
\hline 11 & 50 & 5 & 126 & 85.33 \\
\hline 12 & 52 & 0 & 130.5 & 83.98 \\
\hline
\end{tabular}

Table 1. Technological parameters and the results of $\mathrm{SiO} 2$ coating samples

Coating sample measurement was performed on spectrophotometer MC 122 for light transmission coefficient calculation and FILMETRICS F20-UV machine to determine coating thickness. SiO2 coating light transmission should not be less than $88 \%$ to match the requirements. Results showed that in leak situation under certain circumstances coating light transmission property does not match $88 \%$ boundary. After coating visual verification it was concluded that $\mathrm{SiO} 2$ coated samples No. 4, 5, 6, and 7 are pure, clean and doesn't have any browning signs, thus visual coating quality matches the desired requirements.

\section{Design of prediction models using Fuzzy Logic}

One of the main reasons of the research is to develop prediction models to control $\mathrm{SiO} 2$ coating light transmission coefficient and thickness under leak circumstances. Developed prediction models can be implemented in vacuum coating sputtering laboratories or factories. In the begining input values were defined, then the fuzzification was performed or in other words fuzzy logic was used [4]. The next step included generation of output values according to the defined fuzzy rules previously defined in the database [4]. After fuzzy model was developed, a defuzzification we performed in order to obtain $3 \mathrm{D}$ output parameter plot.

Fuzzy modelling was performed using fuzzyTECH 8.30b Professional Demo software. To develop fuzzy logic model two input variables were used: $\mathrm{O} 2$ flow rate and air flow rate, while output variables were coating light transmission coefficient (T) and thickness (d). The following model shows relationship between input data, output data and rule block (see figures 1 and 2). 


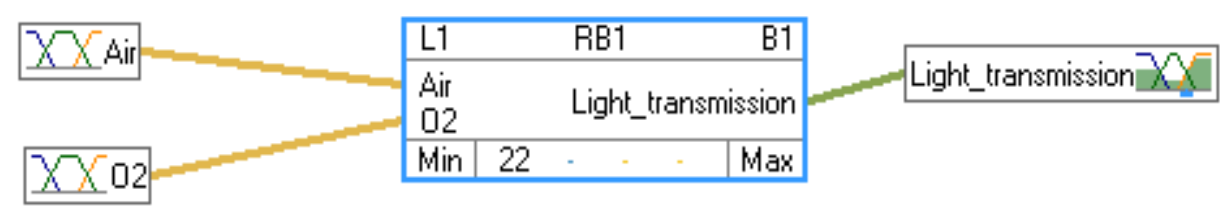

Fig. 1. Light transmission coefficient fuzzy model

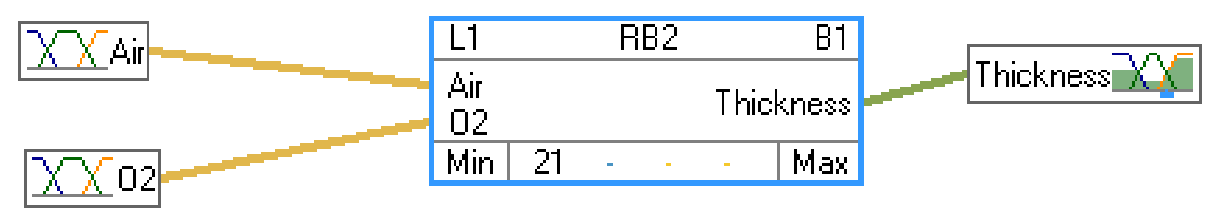

Fig. 2. SiO2 nano coating thickness fuzzy model

After fuzzy logic model development it was necessary to define membership functions to all input and output parameters and create rule block definitions between technological and output parameters. Logic variables have truthvalues that lies in range between 0 and 1. Membership functions for input and output parameters were developed based on the results from experiment conducted and described, thus these results are considered as preliminary result database which is shown in table 1.

For the airflow five membership functions were selected that are as follows (shown at figure 3): very small (12 sccm); small (24 sccm); medium (36 scmm); big (48 sccm); very big $(60 \mathrm{sccm})$.

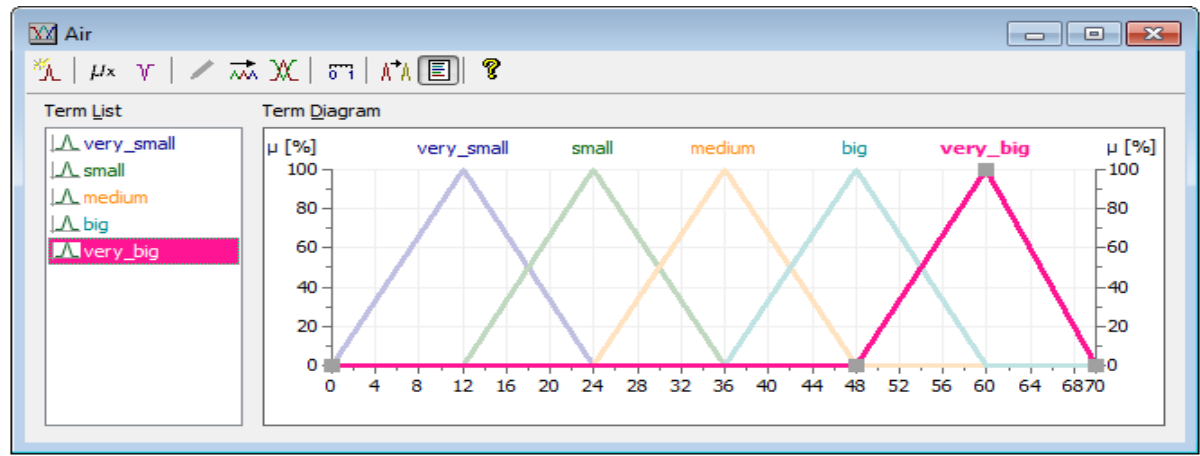

Fig. 3. Five membership functions for air flow rate

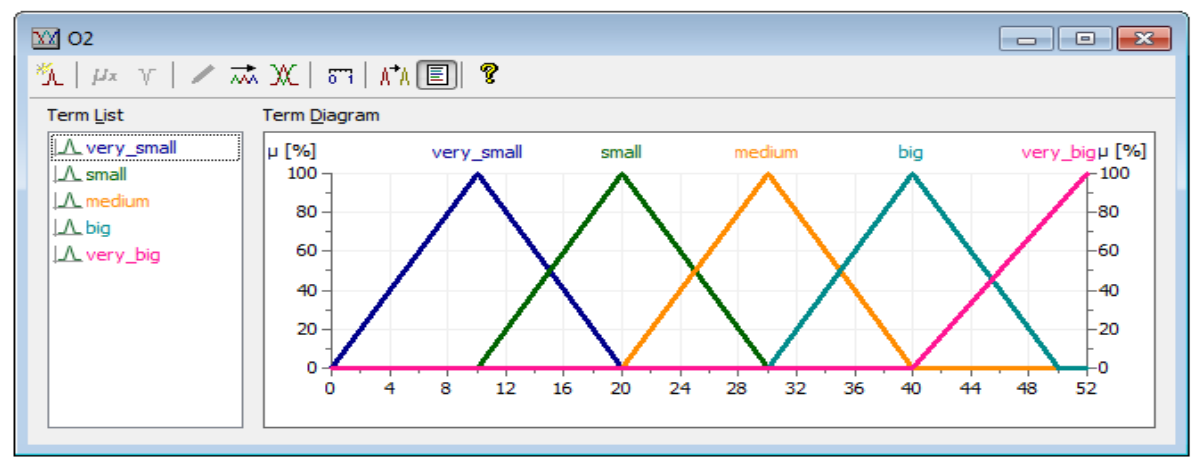

Fig. 4. Five membership functions for $\mathrm{O} 2$ flow rate

For oxygen flow rate (O2) five membership functions were selected that are as follows (shown at figure 4): very small (10 sccm); small (20 sccm); medium (30 sccm); big (40 sccm); very big $(52 \mathrm{sccm})$.

Similar fuzzification procedure was made for thickness and light transmission coefficient (shown at figure 5). For light transmission coefficient five membership functions were selected that are as follows: very small $(84,5 \%)$; small (86\%); medium (87\%); big (88.5\%); very big $(90 \%)$. 


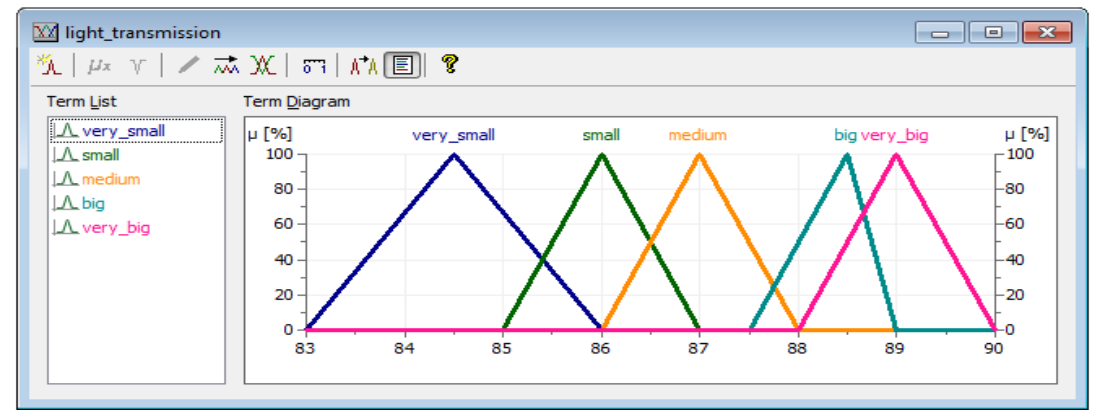

Fig. 5. Five light transmission membership functions

For thickness were selected five membership functions, as follows (shown at figure 6): very small (113 nm); small $(119 \mathrm{~nm})$; medium $(126 \mathrm{~nm})$; big $(133 \mathrm{~nm})$; very big $(142 \mathrm{~nm})$.

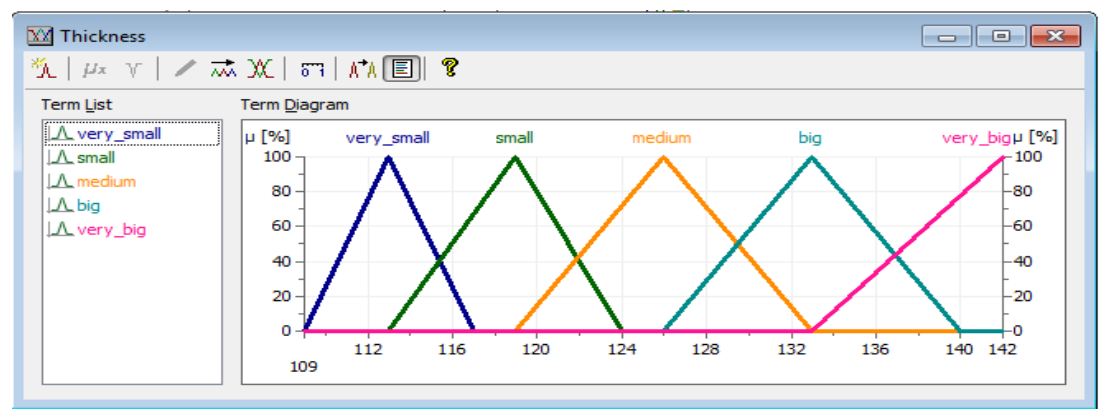

Fig. 6. Five thickness membership functions

The division created by membership function is sufficient to set up a full-fledged light transmission coefficient and thickness fuzzy logic model. Therefore, it is necessary to set a correlation between input and output data using fuzzy rule block, where a database of rules were formed. Since fuzzy logic is based on humanlike operating principles, thus the rule block operates by means of "IF", "THEN". In table 2 a rule block of light transmittance is shown. Rule block database is design based on the obtained and measured $\mathrm{SiO} 2$ coating sputtering experiment results, which are shown in table 1 .

\begin{tabular}{|c|c|c|c|}
\hline & \multicolumn{2}{|c|}{ IF } & THEN \\
\hline Nr. & Air flow & O2 flow & Light transmission coefficient \\
\hline 1 & Very small & Big & Small \\
\hline 2 & Very small & Big & Very small \\
\hline 3 & Very small & Very big & Very small \\
\hline 4 & Very small & Small & big \\
\hline 5 & Very small & Medium & medium \\
\hline 6 & Small & Small & Very big \\
\hline 7 & Small & Medium & Big \\
\hline 8 & Small & Very big & $\mathrm{Big}$ \\
\hline 9 & Small & Very big & medium \\
\hline 10 & Medium & Very small & Big \\
\hline 11 & Medium & Very small & Very big \\
\hline 12 & Medium & Very small & Medium \\
\hline 13 & Medium & Small & Big \\
\hline 14 & Medium & Small & Very big \\
\hline 15 & Medium & Medium & Medium \\
\hline 16 & Big & Very small & Big \\
\hline 17 & $\mathrm{Big}$ & Very small & Medium \\
\hline 18 & Big & Small & Very big \\
\hline 19 & Big & Small & Big \\
\hline 20 & Very big & Very small & Small \\
\hline 21 & Very big & Very small & Medium \\
\hline 22 & Very big & Very small & Big \\
\hline
\end{tabular}

Table 2. Fuzzy logic rule block of light transmission 


\section{Data defuzzification}

Next step after development of the rule block was data defuzzification, where linguistic values are transferred into numerical values and prediction models are graphically displayed in 3D and 2D plots. Light transmission coefficient 3D prediction fuzzy model is shown in figure 7. Thickness 3D prediction fuzzy model is shown in figure 8 . In additional window "Watch: Interactive debug mode" input values can be changed to obtain output values, thus oxygen and air flows can be changed and after data defuzzification thickness and light transmission coefficient values can be obtained.

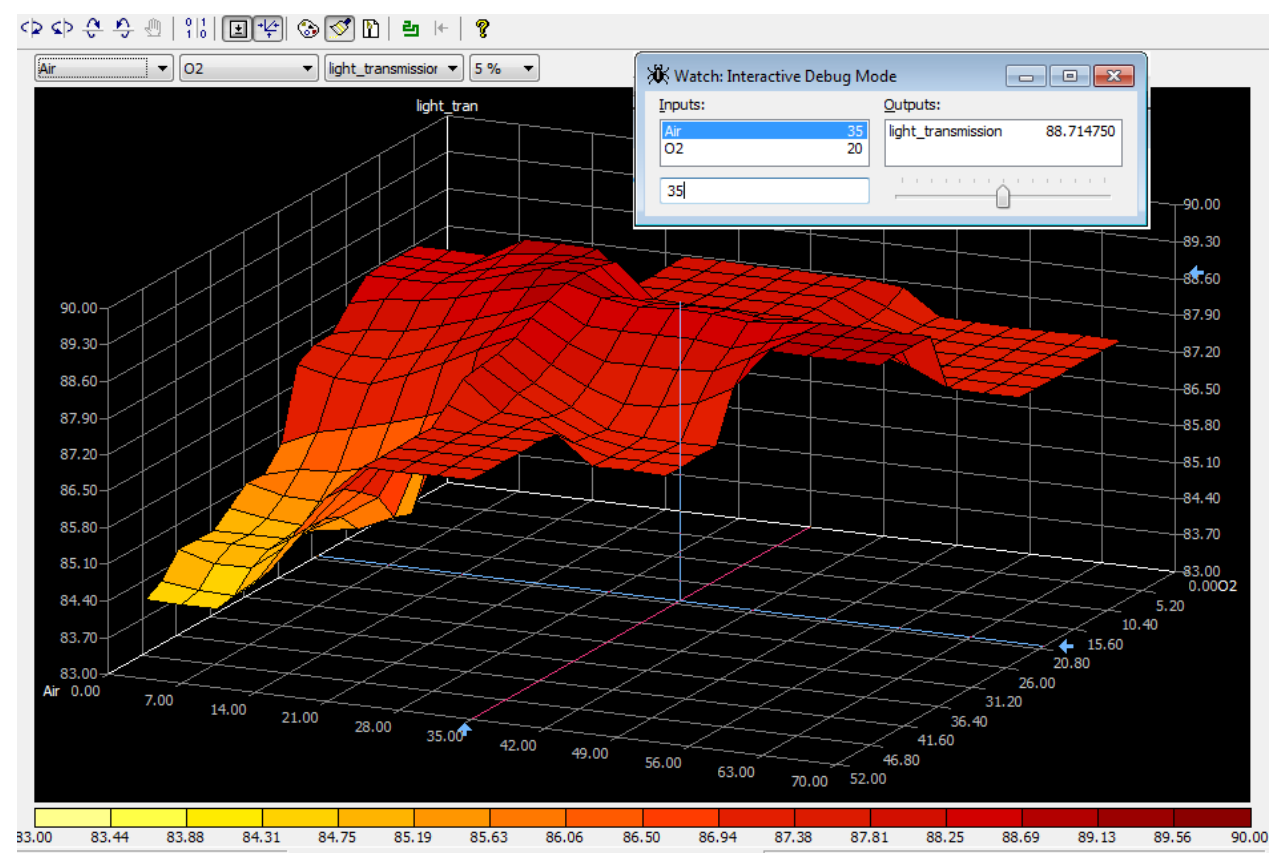

Fig. 7. Light transmission (T) prediction model 3D plot

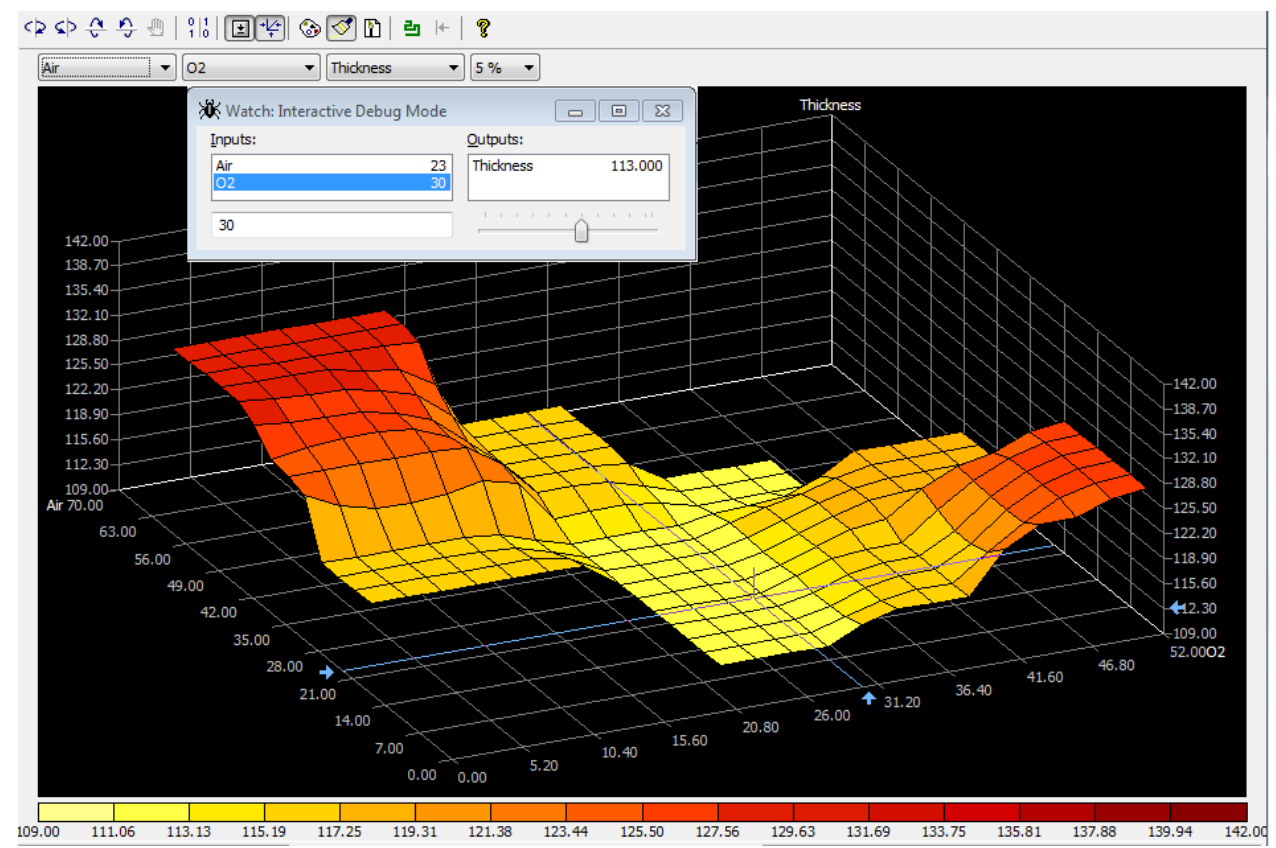

Fig. 8. Coating thickness (d) prediction model 3D plot

One particular example is showed in Fig. 8, where in the interactive debug mode the airflow is to 35 sccm and $\mathrm{O} 2$ flow is $20 \mathrm{sccm}$, thus data defuzzification calculates light transmission coefficient $88.71 \%$. Same principle applies to nano coating thickness prediction model at figure 9, where airflow is $23 \mathrm{sccm}$ and oxygen flow is $30 \mathrm{sccm}$ and thickness prediction model result is $113 \mathrm{~nm}$. Developed fuzzy logic models must be compared with experimental measured results. Therefore, several samples were selected from table 1 and combined with fuzzy logic generated predicted values changing gas flows (see figure 9, 10). 


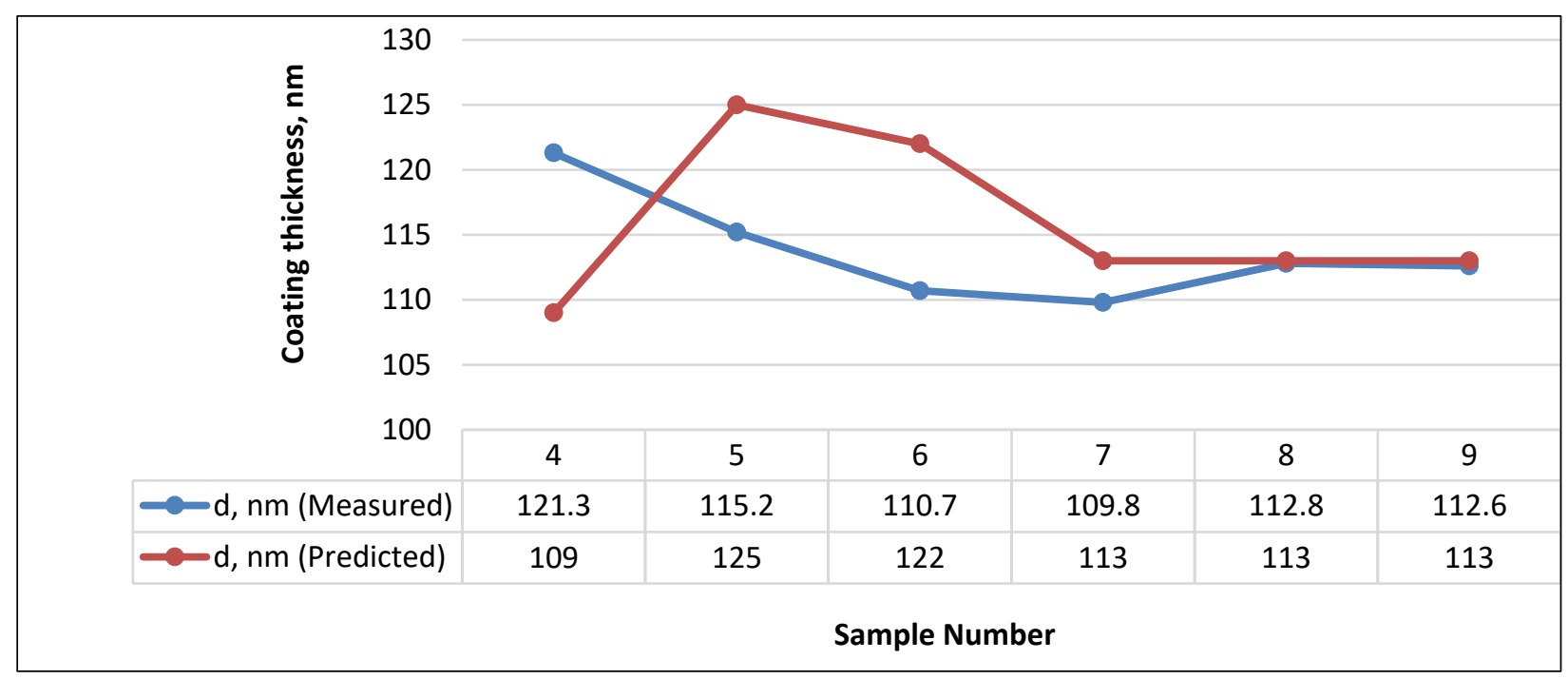

Fig. 9. Measured and predicted nano coating thickness (d)

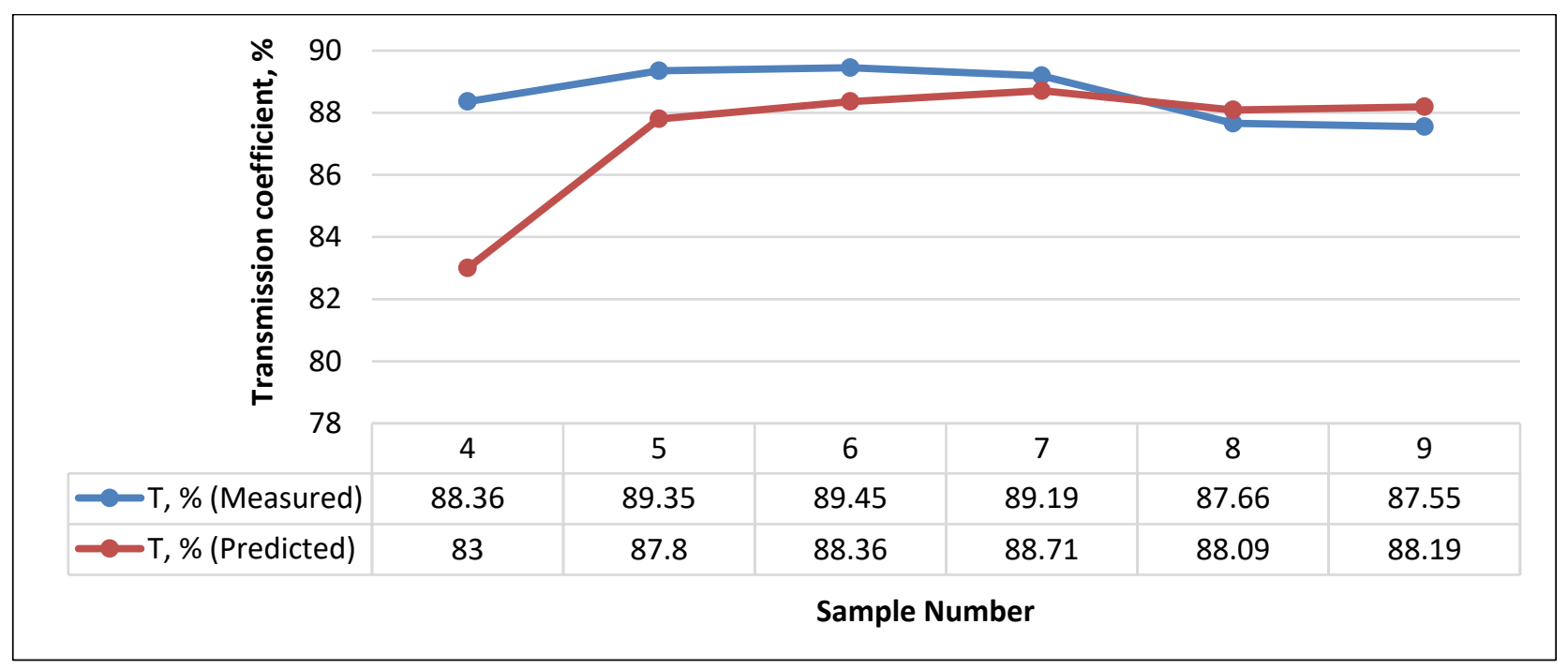

Fig. 10. Measured and predicted light transmission coefficient (T)

Vacuum $\mathrm{SiO} 2$ nano coating light transmission was evaluated as the main criteria, which it must meet. Therefore, if the leak appears it is necessary to follow light transmission prediction model in order to secure determined coating quality. Measured and fuzzy logic calculated light transmission and thickness results are shown in figures 9 and 10. Calculated light transmission results show high accuracy of $93.9 \%$ at fourth sample and highest value of $99.8 \%$ at eight sample. Calculated nano coating thickness results show high accuracy of $90 \%$ at fourth sample and highest value of $99.5 \%$ at eight sample. Light transmission model has high accuracy and can be used for vacuum nano coating properties prediction. Thickness model showed accuracy deviation is from $90 \%$ up to $99.5 \%$. Thus, thickness model accuracy can be considered as decently high. Both models can be used to predict nano coating properties if a leak in vacuum system appears during technological process. As for the priority, the light transmission model remains as main.

\section{Conclusion}

This paper, investigate leak influence on coating quality and its properties during vacuum coating sputtering technological process. Experiment results and developed fuzzy logic models show that it is possible to predict vacuum nano coating light transmission coefficient and thickness under leak conditions with decently high accuracy. Experiments showed that light transmission accuracy diapason is from $93.9 \%$ up to $99.8 \%$ and thickness accuracy diapason is from $90 \%$ up to $99.5 \%$. Fuzzy logic developed models present reliable accuracy, thus can be used in laboratory or vacuum coating sputtering facilities to predict coating properties and quality in leak conditions. Prediction models can save time, material and allow coating process to continue. Fuzzy model development is a complicated task, where precise definition of membership functions and rules are important. 


\section{References}

[1] Ata, S. \& Dincer, K. (2017). Fuzzy logic modeling of performance proton exchange membrane fuel cell with spin method coated with carbon nanotube", International Journal of Hydrogen Energy, Vol. 42, 2017, pp. 2626-2635., https://doi.org/10.1016/j.ijhydene.2016.04.134

[2] Frach, P., Bartzsch, H., Glöß, D., Fahland, M. \& Händel, F. (2008). Electrically insulating Al2O3 and SiO2 films for sensor and photovoltaic applications deposited by reactive pulse magnetron sputtering, hollow cathode arc activated deposition and magnetron-PECVD”, Surface and Coating Techonology, Vol. 202, 2008, pp. 5680-5683., https://doi.org/10.1016/j.surfcoat.2008.06.043

[3] Harun, T., Cemalettin, K., Özer, U. \& Seher, A. (2006). FUZZYFCC: Fuzzy logic control of a fluid catalytic cracking unit (FCCU) to improve dynamic performance, Computers \& Chemical Engineering, Vol. 30/5, 2006, pp. 850863., https://doi.org/10.1016/j.compchemeng.2005.12.016

[4] Kromanis, A. \& Krizbergs, J (2013). Prediction of 3D Surface Roughness Using Regression Analysis and Fuzzy Logic, and their Comparative Analysis, Proceedings of the 20th International DAAAM Symposium 2009, Vienna, Austria, 2528 November 2009, ISSN: 17269679, ISBN: 978-390150970-4, Danube Adria Association for Automation and Manufacturing, DAAAMTop of Form, pp. 803-804.

[5] Mohammad Hossein Nadian., Mohammad Hossein Abbaspour-Fard., Alex Martynenko., Mahmood Reza Golzarian (2017). An intelligent integrated control of hybrid hot air-infrared dryer based on fuzzy logic and computer vision system, Computers and Electronics in Agriculture, Vol. 137, 2017, pp. 138-149., DOI: https://doi.org/10.1016/j.compag.2017.04.001

[6] Ooi, E., Sayuti, M. \& Ahmed A.D.S (2015). Fuzzy logic-based approach to investigate the novel uses of nano suspended lubrication in precise machining of aerospace AL tempered grade 6061, Journal of Cleaner Production, Vol. 89, 2015, pp. 286-295., DOI: https://doi.org/10.1016/j.jclepro.2014.11.006.

[7] Zalnezhad, E. \& Ahmed, A.D.S. (2014). A Fuzzy logic predictive model for better surface roughness of Ti-TiN coating on AL7075-T6 alloy for longer fretting fatigue life", Measurement. Vol. 49, 2014, pp. 256-265., https://doi.org/10.1016/j.measurement.2013.11.042

[8] http://www.sidrabe.com/assets/files/Processes\%20Control\%20for\%20Oxide\%20Layer\%20Deposition\%20in\%20 Roll\%20Vacuum\%20Machines_2013.pdf, (2013). Sidrabe, Publications, Accessed on: 08.06.2017

Roll-to- 